\title{
Charge density waves and surface Mott insulators for adlayer structures on semiconductors: extended Hubbard modeling
}

\author{
Giuseppe Santoro $^{1,2}$, Sandro Scandolo ${ }^{1,2}$, and Erio Tosatti ${ }^{1,2,3}$ \\ (1) International School for Advanced Studies (SISSA), Via Beirut 2, Trieste, Italy \\ (2) Istituto Nazionale per la Fisica della Materia (INFM), Via Beirut 2, Trieste, Italy \\ (3) International Center for Theoretical Physics (ICTP), Strada Costiera, Trieste, Italy
}

\begin{abstract}
Motivated by the recent experimental evidence of commensurate surface charge-density-waves $(\mathrm{CDW})$ in $\mathrm{Pb} / \mathrm{Ge}(111)$ and $\mathrm{Sn} / \mathrm{Ge}(111) \sqrt{ } 3$-adlayer structures, as well as by the insulating states found on $\mathrm{K} / \mathrm{Si}(111): \mathrm{B}$ and $\mathrm{SiC}(0001)$, we have investigated the role of electron-electron interactions, and also of electron-phonon coupling, on the narrow surface state band originating from the outer dangling bond orbitals of the surface. We model the $\sqrt{ } 3$ dangling bond lattice by an extended two-dimensional Hubbard model at half-filling on a triangular lattice. The hopping integrals are calculated by fitting first-principle results for the surface band. We include an on-site Hubbard repulsion $U$ and a nearest-neighbor Coulomb interaction $V$, plus a long-ranged Coulomb tail. The electron-phonon interaction is treated in the deformation potential approximation. We have explored the phase diagram of this model including the possibility of commensurate $3 \times 3$ phases, using mainly the Hartree-Fock approximation. For $U$ larger than the bandwidth we find a non-collinear antiferromagnetic SDW insulator, possibly corresponding to the situation on the $\mathrm{SiC}$ and $\mathrm{K} / \mathrm{Si}$ surfaces. For $U$ comparable or smaller, a rich phase diagram arises, with several phases involving combinations of charge and spin-density-waves (SDW), with or without a net magnetization. We find that insulating, or partly metallic $3 \times 3 \mathrm{CDW}$ phases can be stabilized by two different physical mechanisms. One is the inter-site repulsion $V$, that together with electron-phonon coupling can lower the energy of a charge modulation. The other is a novel magnetically-induced Fermi surface nesting, stabilizing a net cell magnetization of $1 / 3$, plus a collinear SDW, plus an associated weak CDW. Comparison with available experimental evidence, and also with first-principle calculations is made.
\end{abstract}

\section{INTRODUCTION}

$\mathrm{Pb}$ and $\mathrm{Sn} \sqrt{3} \times \sqrt{3}$ adlayer structures on the (111) surface of Ge have recently revealed a reversible charge density wave $(\mathrm{CDW})$ transition to a low temperature reconstructed $3 \times 3$ phase. 1 A half-filled surface state band makes the high temperature phase metallic. The low temperature phase is either metallic - as seems to be the case for $\mathrm{Sn} / \mathrm{Ge}(111)$ - or weakly gapped, or pseudo-gapped, as suggested for $\mathrm{Pb} / \mathrm{Ge}(111)$.

Related isoelectronic systems, like the $\sqrt{3}$-adlayer of $\mathrm{Si}$ on the (0001) surface of $\mathrm{Sid}$ and on $\mathrm{K} / \mathrm{Si}(111)$ : $\mathrm{B}, 8$ show a clear insulating behavior, with a large gap, no structural anomalies, no CDWs, and no transitions, at least to our present knowledge.

These adsorbate surfaces are altogether mysterious. The very existence of a $\sqrt{3} \times \sqrt{3}$ adsorbate phase, with coverage $1 / 3$, is puzzling. For isoelectronic $\mathrm{Si}$ on $\mathrm{Si}(111)$, or $\mathrm{Ge}$ on $\mathrm{Ge}(111)$, for instance, there exists no such phase. The stable low-coverage phase are $7 \times 7$ and $c(2 \times 8)$ respectively, whose coverage is instead close to $1 / 4$. They are made up of $2 \times 2$ basic building blocks, each with one adatom saturating three out of four first-layer atoms, and one unsaturated first-layer atom, the "restatom". In this adatom-restatom block, the nominally unpaired electron of the adatom and that of the restatom pair off together, giving rise to a stable, fully saturated, insulating surface. By contrast, the $\sqrt{3} \times \sqrt{3}$ phases with $1 / 3$ coverage are very common for trivalent adsorbates, such as Ga and In, and for pentavalent ones like As, on the same (111) surfaces. These adatoms lack the unpaired electron, and can therefore lead to a fully saturated insulating surface without the need for any restatoms.

A $\sqrt{3} \times \sqrt{3}$ adsorbate phase of tetravalent adatoms is bound by construction to possess one unpaired electron per adatom, giving rise to a very destabilizing half-filled metallic surface state band. Seen in this crude light, it is a puzzle why this kind of coverage should constitute even only a locally stable state of the surface.

Looking closely, we may speculate that $\mathrm{SiC}(0001)$ 国 and $\mathrm{K} / \mathrm{Si}(111)$ :B, insulators, 10 2 2 are perhaps "stabilized" by Coulomb repulsions, so large to make it anyway difficult for electrons to move. For the more innocent-looking, less correlated, $\mathrm{Pb} / \mathrm{Ge}(111)$ and $\mathrm{Sn} / \mathrm{Ge}(111)$, this argument is less obvious, and the puzzle remains. The function of the $3 \times 3 \mathrm{CDW}$ state - whatever its real nature - most likely serves the function of stabilizing these otherwise unstable surfaces at low temperatures. Nonetheless, the CDW periodicity chosen by 
the surface CDW $-3 \times 3$, meaning a $\sqrt{3} \times \sqrt{3}$ super-cell of adatoms - is not at all evident. In fact, it replaces a supposedly unstable state characterized by an odd number of electrons/cell (three), with another where the electron number (nine) is, alas, odd again.

Be all that as it may, there is little doubt that the main factor driving the phenomena on all these surfaces, appears to be precisely the half-filled- and extremely narrow - surface state band. We thus begin with a discussion that in principle encompasses all the $\sqrt{3} \times \sqrt{3}$ tetravalent adsorbed surfaces.

We believe the following points to be of general validity:

i) Poor nesting.

Two-dimensional Fermi surface (FS) nesting in the half-filled surface states 13 has been repeatedly inyoked as the

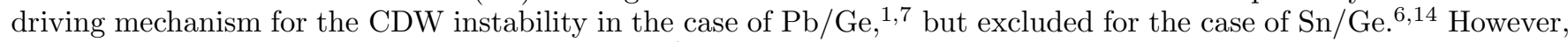
by inspecting either photoemission $k(E)$ data, 35 and existing first-principle (LDA) calculation 1.15 .6 of the surface half-filled band (the "adatom dangling bond band"), we fail to detect a particularly good nesting of the two-dimensional FS at the surface Brillouin zone (BZ) corner $\mathbf{K}=(4 \pi / 3 a, 0)$. The wavevector-dependent susceptibility generated by the calculated band structure, in particular, has no especially large value at this k-point, and rather peaks elsewhere (see inset in Fig. 11). To be sure, there is nothing preventing in general a good nesting at $\mathbf{K}=(4 \pi / 3 a, 0)$, or any other k-point. However, insofar as the surface state band is really lying in a bulk gap at each single k-point, it should be with good accuracy - by simple state counting and charge neutrality - precisely half filled. This implies that the filled and empty state areas should be equal. Hypothetical Fermi surfaces with this kind of shape and good nesting at $\mathbf{K}=(4 \pi / 3 a, 0)$ do not appear to be compatible with an integer electron number. We thus believe lack of perfect nesting to be the case for both $\mathrm{Pb} / \mathrm{Ge}$ as for $\mathrm{Sn} / \mathrm{Ge}$.

Fig. 1. showing a tight binding fit to the LDA surface band dispersion for the test-case of $\mathrm{Si}(111) / \mathrm{Si} 1 \mathrm{1}$ as well as the corresponding FS and Lindhard density response function $\chi_{o}(\mathbf{q})$,

$$
\chi_{o}(\mathbf{q})=\int_{\mathbf{B Z}} \frac{\mathbf{d k}}{(\mathbf{2} \pi)^{2}} \frac{\mathbf{n}_{\mathbf{k}}-\mathbf{n}_{\mathbf{k}+\mathbf{q}}}{\epsilon_{\mathbf{k}+\mathbf{q}}-\epsilon_{\mathbf{k}}},
$$

$n_{\mathbf{k}}$ and $\epsilon_{\mathbf{k}}$ being the occupation number and energy of an electron with Bloch momentum $\mathbf{k}$, provides a concrete illustration of these statements. We note, in passing, that a strong nesting at $\mathbf{K}$ is, on the contrary, automatically guaranteed if the surface band acquires a uniform magnetization in such a way that the densities of up and down electrons become, respectively, $2 / 3$ and $1 / 3.15$ The majority spins would then fill the region external to the reduced BZ in Fig. 1, and their FS would be strongly nested. This suggestion, which turns out to be correct at the mean-field level, points into the direction of a possible role played by magnetism in these systems.

ii) Importance of electron-electron interactions.

The width $W$ of the surface band is relatively small: $W \approx 0.5 \mathrm{eV}$ for $\mathrm{Pb}$ and $\mathrm{Sn} / \mathrm{Ge}(111), W \approx 0.3 \mathrm{eV}$ for $\mathrm{SiC}(0001)$. Moreover, this band is half-filled. These facts call for a careful consideration of electron-electron interactions, as well as of electron-phonon (e-ph), as possible sources of instability. The importance of electron-electron interaction is underlined by the different phenomenology of $\mathrm{SiC}(0001)$ and $\mathrm{K} / \mathrm{Si}(111)$ : $\mathrm{B}$ with respect to $\mathrm{Pb}-\mathrm{Sn} / \mathrm{Ge}(111)$. The stronger insulating character of the former surfaces parallels closely their stronger electron-electron repulsions, connected both with more localized surface Wannier functions (see later on), and with reduced screening, due to larger bulk semiconducting gaps.

iii) Weakness of LDA calculations for ground state prediction.

LDA electronic structure calculations - an extremely well tested tool in many areas- are certainly suitable for a weakly interacting system, such as the bulk semiconductor, or a passivated semiconductor surface. They are less reliable, especially when they do not include spin, in predicting the stable state and the instabilities of a narrow band system. For instance, the phenomenology of $\mathrm{SiC}(0001)$ - suggesting a Mott-Hubbard insulator is unreproducible by LDA. The onset of a CDW on $\mathrm{Sn} / \mathrm{Ge}(111)$ is also not predicted by recent LDA calculations.614 While there is no reason to doubt the basic credibility of the one-electron band energies obtained from these Kohn-Sham equations, the mean-field treatment of interactions, the screened local exchange, and especially the neglect of magnetic correlations are the standard source of problems with LDA. As a consequence, it will be necessary to worry more substantially about interactions, and to use methods which, even if mean-field, permit the inclusion of strong correlations, including magnetic effects.

iv) Interaction-driven mechanisms for $3 \times 3 \mathrm{CDW}$ instabilities.

There are several different couplings which the surface electrons, as they hop weakly between a surface adatom site and another, experience and can influence the formation of the CDW, or of an insulating ground state: a) on-site, and nearest-neighbor (n.n.) inter-site electron-electron repulsion; b) on-site effective attraction (negative Hubbard- $U$ term) of electron-phonon origin.

Because of poor nesting, electron-phonon alone is unlikely to drive the $3 \times 3 \mathrm{CDW}$. At weak coupling, the susceptibility peak in Fig. 1 1 would rather drive an incommensurate periodicity. At strong coupling, the frustration associated to the triangular lattice, will favor, in general, a superconducting ground state over a CDW phase (see Appendix). 10 
On the other hand, the electron-electron interaction, both on-site and, independently, nearest neighbor, naturally suggests, as we shall see later, the $3 \times 3$ surface periodicity, which is found experimentally.

The approach we will take is based on an extended Hubbard-Holstein model. It is by necessity a "non-firstprinciple" approach and, as such, has no strong predictive power. However, it is made more realistic by using parameters extracted from first-principle calculations, and we find it very helpful in clarifying the possible scenarios as a function of the strength of electron-electron interactions. Because of this rather qualitative use, we will make no attempt to push the accuracy of treatment of this model to a very high level of sophistication. The basic tool will be the unrestricted Hartree-Fock approximation. Although mean field, it allows magnetic solutions, favored by exchange which is unscreened.

\section{MODEL}

Each tetravalent adatom on a (111) semiconductor surface carries a dangling bond - an unpaired electron in an unsaturated orbital. In the $\sqrt{3} \times \sqrt{3}$ structure, the dangling bonds of the adatoms give rise to a band of surface states which lies in the bulk semiconductor gap. 1.15 By electron counting, such a band is half-filled. Our basic starting peint is the quantitatively accurate surface state band dispersion $\epsilon_{\mathbf{k}}$ which one calculates in gradient-corrected LDA, 0.15 . It is shown in Fig. 1 for the case of $\mathrm{Si} / \mathrm{Si}(111)$. The solid and dashed lines in Fig. 1 are tight-binding fits to the LDA results obtained by including, respectively, up to the $6^{\text {th }}$ and up to the $2^{\text {nd }}$ shell of neighbors. The fit with hopping integrals $t_{1}, t_{2}, \cdots, t_{6}$ is quite good. Less good, but qualitatively acceptable, is the fit obtained using only nearest neighbor (n.n.) and next-nearest neighbor (n.n.n.) hopping integrals $t_{1}$ and $t_{2}$. The Fermi surface (FS) for the half-filled surface band is shown in the upper inset of Fig. 1. It is important to stress that the FS does not show good nesting properties at the wavevector $\mathbf{q}=\mathbf{K}$ (the BZ corner). This feature is shared by all LDA calculations on similar systems 4 . 5 . Albeit small, the bandwidth $W$ of the surface band is much greater than one would predict by a direct overlap of adatom dangling bonds, as the adatoms are very widely apart, for instance about $7 \AA$ on $\operatorname{Ge}(111)$. Hopping is indirect, and takes place from the adatom to the first-layer atoms underneath, from that to a second-layer atom, then again to a first-layer atom underneath the other adatom, and from there finally to other adatom dangling bond. Thus, when expressed in terms of elementary hopping processes between hybrid orbitals, electron hopping between two neighboring adatom dangling bonds is fifth order. As a result, the final dispersion of the surface state band strongly parallels that of the closest bulk band, the valence band. Correspondingly, hybridization effects of the dangling bond orbitals with first, second, and even third, bulk layer orbitals are strong, as shown by the extension into the bulk of the Wannier orbital associated to the LDA surface band (Fig. 2).

In spite of this, we can still associate to every adatom a Wannier orbital and write the effective Hamiltonian for the surface band as follows:

$$
H=\sum_{\mathbf{k}}^{B Z} \sum_{\sigma} \epsilon_{\mathbf{k}} c_{\mathbf{k}, \sigma}^{\dagger} c_{\mathbf{k}, \sigma}+H_{\mathrm{ph}}+H_{\mathrm{e}-\mathrm{ph}}+H_{\mathrm{int}},
$$

where $c_{\mathbf{k}, \sigma}^{\dagger}$ is the Fourier transform of the Wannier orbital, namely the surface state in a Bloch picture. The sum over the wavevectors runs over the surface BZ. $H_{\text {int }}$ includes correlation effects which are not correctly accounted for within LDA, which we parametrize as follows:

$$
H_{\mathrm{int}}=U \sum_{\mathbf{r}} n_{\mathbf{r}, \uparrow} n_{\mathbf{r}, \downarrow}+\frac{1}{2} \sum_{\mathbf{r} \neq \mathbf{r}^{\prime}} V_{\mathbf{r}-\mathbf{r}^{\prime}}\left(n_{\mathbf{r}}-1\right)\left(n_{\mathbf{r}^{\prime}}-1\right) .
$$

Here $U$ is an effective repulsion (Hubbard- $U$ ) for two electrons on the same adatom Wannier orbital, and $V_{\mathbf{r}-\mathbf{r}^{\prime}}$ is the direct Coulomb interaction between different sites $\mathbf{r}$ and $\mathbf{r}^{\prime} .17$ Let $V$ be the n.n. value of $V_{\mathbf{r}-\mathbf{r}^{\prime}}$, which is, clearly, the largest term. We have considered two models for $V_{\mathbf{r}-\mathbf{r}^{\prime}}$ : a model (A) in which we truncate $V_{\mathbf{r}-\mathbf{r}^{\prime}}$ to n.n., and a model (B) in which $V_{\mathbf{r}-\mathbf{r}^{\prime}}$ has a long range Coulombic tail of the form

$$
V_{\mathbf{r}-\mathbf{r}^{\prime}}=\frac{a V}{\left|\mathbf{r}-\mathbf{r}^{\prime}\right|},
$$

where $a$ is the n.n. distance. The results for model B are qualitatively similar to those of A, and will be only briefly discussed later on. In other words, even if most of the detailed results in this paper will be base on the n.n. $V_{\mathbf{r}-\mathbf{r}^{\prime}}$, their validity is more general.

LDA estimates of the bare coulomb repulsion $U_{o}$ and $V_{o}$ between two electrons respectively on the same and on neighboring Wannier orbitals are - for our test case of $\mathrm{Si}(111) / \mathrm{Si}$ - of about $3.6 \mathrm{eV}$ and $1.8 \mathrm{eV}$ respectively. .5 
Screening effects by the the underlying bulk are expected to reduce very substantially these repulsive energies. An order of magnitude estimate for $U$ and $V$ is obtained by dividing their bare values by the image-charge screening factor, $(\epsilon+1) / 2 \approx 6$, yielding, for $\mathrm{Si}, U=0.6 \mathrm{eV}\left(10 t_{1}\right)$, and $\mathrm{G}=0.3 \mathrm{eV}\left(5 t_{1}\right)$. Corresponding values would be somewhat smaller for $\mathrm{Ge}(111)$, in view of a very similar dispersion 14 and of a ratio of about $4 / 3$ between the dielectric consfants of $\mathrm{Ge}$ and $\mathrm{Si}$. $\mathrm{SiC}(0001)$, the opposite is true. The surface state band is extremely narrow, of order 0.3 $\mathrm{eV}$, while the bulk dielectric constant is only about 6.5 .

As for the e-ph interaction, in principle both the on-site Wannier state energy and the hopping matrix elements between neighbors depend on the positions of the adatoms. Within the deformation potential approximation, we consider only a linear dependence of the on-site energy from a single ionic coordinate (for instance, the height $z_{\mathbf{r}}$ of the adatom measured from the equilibrium position), and take

$$
H_{\mathrm{e}-\mathrm{ph}}=-g \sum_{\mathbf{r}} z_{\mathbf{r}}\left(n_{\mathbf{r}}-1\right),
$$

with $g$ of the order of $\approx 1 \mathrm{eV} / \AA$. The free-phonon term will have the usual form

$$
H_{\mathrm{ph}}=\sum_{\mathbf{k}}^{B Z} \hbar \omega_{\mathbf{k}}\left(b_{\mathbf{k}}^{\dagger} b_{\mathbf{k}}+\frac{1}{2}\right),
$$

where $b_{\mathbf{k}}$ is the phonon annihilation operator, and $\hbar \omega_{\mathbf{k}}$ a typical phonon frequency of the system, which we take to be about $30 \mathrm{meV}$, independent of $\mathbf{k}$.

\section{PHASE DIAGRAM: SOME LIMITING CASES}

Preliminary to the full treatment of Sect. IV, we consider first the purely electronic problem in the absence of e-ph interaction. We start the discussion from particular limiting cases for which well-controlled statements, or at least intuitively clear ones, can be made, without the need of any new specific calculations. In the Appendix we will also consider, because it is useful in connection with the electron-phonon case, the unphysical limit of strong on-site attraction (large and negative $U$ ).

\section{A. Large positive $U$ : the Mott insulator.}

For $U \gg V, W$, the system is deep inside the Mott insulating regime 19 The charge degrees of freedom are frozen, with a gap of order U. The only dynamics is in the spin degrees of freedom. Within the large manifold of spin degenerate states with exactly one electron per site, the kinetic energy generates, in second order perturbation theory, a Heisenberg spin-1/2 antiferromagnetic effective Hamiltonian governing the spin degrees of freedom,

$$
H_{\mathrm{eff}}=\sum_{(i j)} J_{i j} \mathbf{S}_{\mathbf{r}_{i}} \cdot \mathbf{S}_{\mathbf{r}_{j}}
$$

with $J_{i j}=4\left|t_{i j}\right|^{2} / U$

For our test case of $\mathrm{Si}(111) / \mathrm{Si}$, the values of the hoppings are such that $J_{1} \approx 20 \mathrm{meV}, J_{2} / J_{1} \approx 0.12$ while the remaining couplings $J_{3}, \cdots$ are very small. Antiferromagnetism is frustrated on the triangular lattice. Zero temperature long range order (LRO) - if present - should be of the three-sublattice $120^{\circ}$-Néel type, which can be also seen as a commensurate spiral spin density wave (s-SDW).

Because it does not imbalance charge, this state is not further affected by electron-phonon coupling.

In summary, we expect for large values of $U$ a wide-gap Mott insulator with a s-SDW (spins lying in a plane, forming $120^{\circ}$ angles), a $3 \times 3$ magnetic unit cell, but uniform charge (no $\mathrm{CDW}$ ). This is, most likely, the state to be found on the Si-terminated and C-terminated $\mathrm{SiC}(0001)$ surface at $\mathrm{T}=011 \mathrm{L2}$.

\section{B. Strong inter-site repulsion: an asymmetric CDW with three inequivalent sites.}

The e-ph coupling can effectively reduce $U$, but not $V$. Therefore, it is of interest to consider the hypothetical regime $W<U \ll V$. When the first-neighbor electron-electron repulsion $V$ is large the system, in order to minimize 
the interaction energy, will prefer a $3 \times 3 \mathrm{CDW}$-like ground state, with two electrons on one sublattice (A), a single electron on another sublattice (B), and zero electrons on the third sublattice (C) (see Fig. 3). These states are still highly degenerate (in the absence of hopping) due to spin degeneracy for the single unpaired electron on sublattice B. A gap $U$ separates these states from the lowest-energy excited configurations (see Fig. 3). The spin degeneracy can be removed in second-order perturbation theory, owing to $t_{2}$, which leads to an effective spin- $1 / 2$ Heisenberg Hamiltonian within sublattice B,

$$
H_{\text {eff }}=J \sum_{(i j)}^{\text {sublattice B }} \mathbf{S}_{\mathbf{r}_{i}} \cdot \mathbf{S}_{\mathbf{r}_{j}},
$$

with a weak antiferromagnetic exchange constant $J=4 t_{2}^{2} / U .19$ Summarizing, we expect in this regime a strong $3 \times 3$ asymmetric CDW (a-CDW) with three inequivalent sites $\left(\phi_{\rho} \approx \pi / 6\right.$, see below), and a spiral $3 \sqrt{3} \times 3 \sqrt{3} \mathrm{SDW}$, governing the unpaired electron spins, superimposed on it. Notice that, while the charge periodicity is $3 \times 3$, the actual unit cell is larger, i.e., $3 \sqrt{3} \times 3 \sqrt{3}$. Despite having the correct charge periodicity, namely $3 \times 3$, this a-CDW is not compatible with the experimental findings on $\mathrm{Pb}-\mathrm{Sn} / \mathrm{Ge}$, which is a symmetric $\mathrm{CDW}$. We conclude that the low-temperature CDW state of these systems is not completely dominated by $V$.

\section{MEAN-FIELD THEORY.}

In order to get a more complete picture of additional phases for smaller $U$, and of the possible phase diagram of the model we now turn to a quantitative mean field theory analysis. The first issue is to include the possibility of magnetic correlations. For small values of the interactions $U$ and $V$, the Stoner criterion can be used to study the possible magnetic instabilities of the paramagnetic metal obtained from LDA calculations. The charge and spin susceptibilities are given, within the random phase approximation, 20 by

$$
\begin{aligned}
& \chi_{C}(\mathbf{q})=\frac{2 \chi_{o}(\mathbf{q})}{1+\left(U+2 V_{\mathbf{q}}\right) \chi_{o}(\mathbf{q})} \\
& \chi_{S}(\mathbf{q})=\frac{\chi_{o}(\mathbf{q})}{1-U \chi_{o}(\mathbf{q})},
\end{aligned}
$$

where $\chi_{o}$ is the non-interacting susceptibility per spin projection, and both factors of 2 account for spin degeneracy. The divergence of $\chi_{S}$ is governed, in this approximation, by $U$ only. Since $\chi_{o}(\mathbf{q})$ is finite everywhere, a finite $U$ is needed in order to destabilize the paramagnetic metal. The wavevector $\mathbf{q}^{*}$ at which $\chi_{S}$ first diverges, by increasing $U$, is in generalincommensurate with the underlying unit cell. The instability is towards an incommensurate, metallic, spiral SDW.21 Fig. 1 shows that, in our case, $\mathbf{q}^{*}=(1.32 K, 0)$ (with $K=4 \pi / 3 a$, the BZ corner). We get $U_{c}^{H F} / t_{1} \approx 3.7$. (The other maximum of $\chi_{o}$ at $\mathbf{q}=(0.525 K, 0)$ is very close to the result obtained for the triangular lattice with n.n. hopping only.21) As for the charge susceptibility, a divergence can be caused only by an attractive Fourier component of the potential $V_{\mathbf{q}}$. $V_{\mathbf{q}}$ has a minimum at the BZ corners $\pm \mathbf{K}$, with $V_{ \pm \mathbf{K}}=-3 V$ for the n.n. model (A) $\left(V_{ \pm \mathbf{K}} \approx-1.5422 \mathrm{~V}\right.$ if a Coulomb tail is added, model B). This minimum leads to an instability towards a $3 \times 3$ CDW as $\left(U+2 V_{\mathbf{K}}\right) \chi_{o}(\mathbf{K})=-1$, i.e., given our value of $\chi_{o}(\mathbf{K}) \approx 0.2 / t_{1},\left(U+2 V_{\mathbf{K}}\right) \approx-5 t_{1}$. For model A we get a transition, when $U=0$, at $V_{c}^{M F} / t_{1} \approx 0.83$.

In general, the small coupling paramagnetic metal is surrounded by an intermediate coupling region, where complicated incommensurate - generally metallic - solutions occur. For stronger $U$ and $V$, commensurate solutions are privileged 21 In view of the fact that a $3 \times 3 \mathrm{CDW}$ is experimentally relevant, we concentrate our analysis on the simplest commensurate phases. These are easy to study with a standard Hartree-Fock (HF) mean-field theory. In particular, we restrict ourselves to order parameters associated with non-vanishing momentum space averages of the type $\left\langle c_{\mathbf{k}, \sigma}^{\dagger} c_{\mathbf{k}, \sigma^{\prime}}\right\rangle$ and $\left\langle c_{\mathbf{k}, \sigma}^{\dagger} c_{\mathbf{k} \pm \mathbf{K}, \sigma^{\prime}}\right\rangle$. Possible non-vanishing order parameters are the uniform magnetization density $\mathbf{m}$,

$$
\mathbf{m}=\frac{1}{N_{s}} \sum_{\mathbf{k}}^{B Z} \sum_{\alpha, \beta}\left\langle c_{\mathbf{k}, \alpha}^{\dagger}(\vec{\sigma})_{\alpha \beta} c_{\mathbf{k}, \beta}\right\rangle=\frac{2}{N_{s}}\left\langle\mathbf{S}_{\text {tot }}\right\rangle,
$$

the $\mathbf{K}$-component of the charge density,

$$
\rho_{\mathbf{K}}=\frac{1}{N_{s}} \sum_{\mathbf{k}}^{B Z} \sum_{\sigma}\left\langle c_{\mathbf{k}, \sigma}^{\dagger} c_{\mathbf{k}-\mathbf{K}, \sigma}\right\rangle,
$$


and the $\mathbf{K}$-component of the spin density

$$
\mathbf{S}_{\mathbf{K}}=\frac{1}{N_{s}} \sum_{\mathbf{k}}^{B Z} \sum_{\alpha, \beta}\left\langle c_{\mathbf{k}, \alpha}^{\dagger} \frac{(\vec{\sigma})_{\alpha \beta}}{2} c_{\mathbf{k}-\mathbf{K}, \beta}\right\rangle .
$$

Note that only $\rho_{\mathbf{K}}$ and $\mathbf{S}_{\mathbf{K}}$ are $3 \times 3$ periodic. Moreover, $K$-components of bond order parameters of the type $\left\langle c_{\mathbf{r}, \sigma}^{\dagger} c_{\mathbf{r}^{\prime}, \sigma^{\prime}}\right\rangle$ are automatically included in the calculation. $\rho_{\mathbf{K}}$ and $\mathbf{S}_{\mathbf{K}}$ have phase freedom, and are generally complex: $\rho_{\mathbf{K}}=\left|\rho_{\mathbf{K}}\right| e^{i \phi_{\rho}}$, etc. The role of the phase is clarified by looking at the real-space distribution within the $3 \times 3$ unit cell. For the charge, for instance, $\left\langle n_{\mathbf{r}_{j}}\right\rangle=1+2\left|\rho_{\mathbf{K}}\right| \cos \left(2 \pi p_{j} / 3+\phi_{\rho}\right)$, where $p_{j}=0,1,2$, respectively, on sublattice A, $\mathrm{B}$, and $\mathrm{C}$. The e-ph coupling is included but, after linearization, the displacement order parameter is not independent, and is given by $\left\langle z_{\mathbf{K}}\right\rangle=\left(g / M \omega_{\mathbf{K}}^{2}\right) \rho_{\mathbf{K}}$. Only the phonon modes at $\pm \mathbf{K}$ couple directly to the CDW. The phonon part of the Hamiltonian can be diagonalized by displacing the oscillators at $\pm \mathbf{K}$. This gives just an extra term in the electronic HF Hamiltonian of the form $\Delta U\left(\rho_{\mathbf{K}}^{*} \hat{\rho}_{\mathbf{K}}+\right.$ H.c. $)$, with an energy $\Delta U=-g^{2} / M \omega_{\mathbf{K}}^{2}$ which is the relevant coupling parameter. This term acts, effectively, as a negative- $U$ contribution acting only on the charge part of the electronic Hamiltonian.

With the previous choice of non-vanishing momentum space averages, the Hartree-Fock Hamiltonian reads:

$$
\begin{aligned}
H_{\mathrm{H}-\mathrm{F}}= & \sum_{\mathbf{k}}^{B Z} \sum_{\sigma} \epsilon_{\mathbf{k}} n_{\mathbf{k}, \sigma}-U \mathbf{m} \cdot \mathbf{S}_{\mathrm{tot}} \\
& +\sum_{\mathbf{k}}^{B Z} \sum_{\sigma}\left\{\left[\left(\frac{U}{2}+V_{\mathbf{K}}-\frac{g^{2}}{M \omega_{\mathbf{K}}^{2}}\right) \rho_{\mathbf{K}}-\sigma U S_{\mathbf{K}}^{z}\right] c_{\mathbf{k}, \sigma}^{\dagger} c_{\mathbf{k}+\mathbf{K}, \sigma}+\text { H.c. }\right\} \\
& -U \sum_{\mathbf{k}}^{B Z}\left\{S_{\mathbf{K}}^{+} c_{\mathbf{k}, \downarrow}^{\dagger} c_{\mathbf{k}+\mathbf{K}, \uparrow}+S_{\mathbf{K}}^{-} c_{\mathbf{k}, \uparrow}^{\dagger} c_{\mathbf{k}+\mathbf{K}, \downarrow}+\text { H.c. }\right\} \\
& +\sum_{\mathbf{k}}^{B Z} \sum_{\sigma}\left\{A_{\mathbf{k}}^{(\sigma \sigma)} c_{\mathbf{k}, \sigma}^{\dagger} c_{\mathbf{k}, \sigma}+\left[B_{\mathbf{k}}^{(\sigma \sigma)} c_{\mathbf{k}, \sigma}^{\dagger} c_{\mathbf{k}+\mathbf{K}, \sigma}+\text { H.c. }\right]\right\} \\
& +\sum_{\mathbf{k}}^{B Z} \sum_{\sigma}\left\{A_{\mathbf{k}}^{(\bar{\sigma} \sigma)} c_{\mathbf{k}, \bar{\sigma}}^{\dagger} c_{\mathbf{k}, \sigma}+\left[B_{\mathbf{k}}^{(\bar{\sigma} \sigma)} c_{\mathbf{k}, \bar{\sigma}}^{\dagger} c_{\mathbf{k}+\mathbf{K}, \sigma}+\text { H.c. }\right]\right\}
\end{aligned}
$$

The last two terms originate exchange contributions due to the $V$-term; $A_{\mathbf{k}}^{\left(\sigma^{\prime} \sigma\right)}$ and $B_{\mathbf{k}}^{\left(\sigma^{\prime} \sigma\right)}$ are shorthands for the following convolutions:

$$
\begin{aligned}
& A_{\mathbf{k}}^{\left(\sigma^{\prime} \sigma\right)}=-\frac{1}{N_{s}} \sum_{\mathbf{k}^{\prime}}^{B Z} V_{\mathbf{k}-\mathbf{k}^{\prime}}\left\langle c_{\mathbf{k}^{\prime}, \sigma}^{\dagger} c_{\mathbf{k}^{\prime}, \sigma^{\prime}}\right\rangle \\
& B_{\mathbf{k}}^{\left(\sigma^{\prime} \sigma\right)}=-\frac{1}{N_{s}} \sum_{\mathbf{k}^{\prime}}^{B Z} V_{\mathbf{k}-\mathbf{k}^{\prime}}\left\langle c_{\mathbf{k}^{\prime}+\mathbf{K}, \sigma^{\dagger}}^{\dagger} c_{\mathbf{k}^{\prime}, \sigma^{\prime}}\right\rangle .
\end{aligned}
$$

The BZ is divided into three regions: a reduced BZ (RBZ), and the two zones obtained by $\mathbf{k} \pm \mathbf{K}$ with $\mathbf{k} \in \mathrm{RBZ}$. The HF problem in Eq. 11 reduces to the self-consistent diagonalization of a $6 \times 6$ (including the spin) matrix for each $\mathbf{k} \in \mathrm{RBZ}$.

\section{A. Landau theory}

The mean-field solutions must be compatible with the symmetry of the problem. Before discussing the HF phase diagram we obtain, it is useful to present a few general phenomenological considerations based on a symmetry analysis of the Landau theory built from the CDW order parameter $\rho_{\mathbf{K}}$ (a complex scalar), the SDW order parameter $\mathbf{S}_{\mathbf{K}}$ (a complex vector), and the uniform magnetization $\mathbf{m}$ (a real vector).22 In the absence of spin-orbit coupling, the possible contributions to the Laundau free energy $F$ allowed by symmetry, up to fourth order, have the form

$$
F=\frac{1}{2} a_{\rho}\left|\rho_{\mathbf{K}}\right|^{2}+\frac{1}{2} a_{m}|\mathbf{m}|^{2}+\frac{1}{2} a_{s}\left|\mathbf{S}_{\mathbf{K}}\right|^{2}+F_{3}+F_{4}
$$




$$
\begin{aligned}
F_{3}= & \left(B_{\rho} \rho_{\mathbf{K}}^{3}+\text { c.c. }\right)+\left[B_{\rho s} \rho_{\mathbf{K}}\left(\mathbf{S}_{\mathbf{K}} \cdot \mathbf{S}_{\mathbf{K}}\right)+\text { c.c. }\right] \\
F_{4}= & b_{\rho}\left|\rho_{\mathbf{K}}\right|^{4}+b_{m}|\mathbf{m}|^{4}+b_{s}^{(1)}\left|\mathbf{S}_{\mathbf{K}}\right|^{4}+b_{s}^{(2)}\left(\mathbf{S}_{\mathbf{K}} \times \mathbf{S}_{\mathbf{K}}^{*}\right)^{2} \\
& +b_{\rho s}\left|\rho_{\mathbf{K}}\right|^{2}\left|\mathbf{S}_{\mathbf{K}}\right|^{2}+b_{\rho m}\left|\rho_{\mathbf{K}}\right|^{2}|\mathbf{m}|^{2}+b_{m s}^{(1)}|\mathbf{m}|^{2}\left|\mathbf{S}_{\mathbf{K}}\right|^{2}+b_{m s}^{(2)}\left(\mathbf{m} \cdot \mathbf{S}_{\mathbf{K}}\right)\left(\mathbf{m} \cdot \mathbf{S}_{\mathbf{K}}^{*}\right) \\
& +\left[b_{m s}^{(3)}\left(\mathbf{m} \cdot \mathbf{S}_{\mathbf{K}}\right)\left(\mathbf{S}_{\mathbf{K}} \cdot \mathbf{S}_{\mathbf{K}}\right)+\text { c.c. }\right]+\left[b_{\rho m s} \rho_{\mathbf{K}}^{2}\left(\mathbf{m} \cdot \mathbf{S}_{\mathbf{K}}\right)+\text { c.c. }\right],
\end{aligned}
$$

with $\left|\mathbf{S}_{\mathbf{K}}\right|^{2}=\left(\mathbf{S}_{\mathbf{K}} \cdot \mathbf{S}_{\mathbf{K}}^{*}\right)$. Notice that third order invariants are present due to commensurability, $3 \mathbf{K}=\mathbf{G}$ (reciprocal lattice vector). Therefore, first order transitions are generally possible.22

This expansion suggests a number of additional comments: i) A CDW can occur without accompanying magnetism, i.e., $\rho_{\mathbf{K}} \neq 0$, while $\mathbf{m}=0$ and $\mathbf{S}_{\mathbf{K}}=0$. This is the case, as we shall see later, for the small $U$ region of the HF phase diagram. ii) The possible SDW phases are either collinear (l-SDW) (for which $\left(\mathbf{S}_{\mathbf{K}} \times \mathbf{S}_{\mathbf{K}}^{*}\right)=0$ ) or coplanar.23 The latter have, with a suitable choice of the phases, $\mathbf{S}_{\mathbf{K}}^{x}=\left|\mathbf{S}_{\mathbf{K}}\right| \cos \alpha$ and $\mathbf{S}_{\mathbf{K}}^{y}=-i\left|\mathbf{S}_{\mathbf{K}}\right| \sin \alpha$, and can be generally described as a spiral SDW (s-SDW)

$$
\left\langle\mathbf{S}_{\mathbf{r}}\right\rangle=2\left|\mathbf{S}_{\mathbf{K}}\right|[\hat{\mathbf{x}} \cos \alpha \cos (\mathbf{K} \cdot \mathbf{r})-\hat{\mathbf{y}} \sin \alpha \sin (\mathbf{K} \cdot \mathbf{r})],
$$

with an eccentricity parameter $\alpha \neq 0, \pi / 2$. ( $\alpha=0$ or $\pi / 2$ are actually l-SDW along the $\hat{\mathbf{x}}$ or $\hat{\mathbf{y}}$ directions.) $\alpha=\pi / 4$ describes a circular spiral SDW. Now, the only possibility of having a SDW without CDW is via a circular spiral SDW $(\alpha=\pi / 4)$. Indeed, the third order invariant $\left[B_{\rho s} \rho_{\mathbf{K}}\left(\mathbf{S}_{\mathbf{K}} \cdot \mathbf{S}_{\mathbf{K}}\right)+\right.$ c.c. $]$ vanishes by symmetry only for a circular spiral SDW, for which $\left(\mathbf{S}_{\mathbf{K}} \cdot \mathbf{S}_{\mathbf{K}}\right)=0$; in all other cases, a SDW implies - if $B_{\rho s} \neq 0-$ a CDW as well. iii) The simultaneous presence of a SDW and a CDW implies, generally, a finite magnetization $\mathbf{m}$, via the fourth order invariant $\left[b_{\rho m s} \rho_{\mathbf{K}}^{2}\left(\mathbf{m} \cdot \mathbf{S}_{\mathbf{K}}\right)+\right.$ c.c.], unless the phases of $\rho$ and $S$ are such that $2 \phi_{\rho}+\phi_{\sigma}=\pi / 2+n \pi$. This happens in phase $\mathrm{E}$ of our phase diagram, which has therefore no uniform magnetization. $i v)$ The presence of a SDW leads, generally, to a finite uniform magnetization as well, via the fourth order invariant $\left[b_{m s}^{(3)}\left(\mathbf{m} \cdot \mathbf{S}_{\mathbf{K}}\right)\left(\mathbf{S}_{\mathbf{K}} \cdot \mathbf{S}_{\mathbf{K}}\right)+\right.$ c.c. $]$, unless the phase $\phi_{\sigma}$ is such that $3 \phi_{\sigma}=\pi / 2+m \pi$.

\section{B. Phase diagram in the Hartree-Fock approximation}

We present a brief summary of the mean-field HF calculations for arbitrary $U, V$, and $g$, obtained by solving numerically the self-consistent problem in Eqs. 8 12. The main phases present in the HF phase diagram are shown in Fig. 1 for the case of $g=0$. The effect of $g \neq 0$ will be discussed further below.

Phase A: Spiral SDW insulating phase. The circular spiral SDW (phase A) dominates the large $U$, small $V$ part of the phase diagram, as expected from the Heisenberg model mapping at $U \rightarrow \infty$ (see sect. III A). This is the

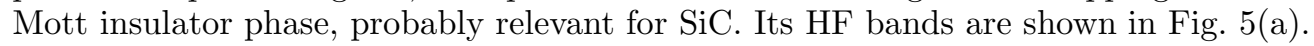

Phase A': Collinear SDW with $m^{z}=1 / 3$ insulating phase. This is another solution of the HF equations in the large $U$, small $V$ region. It is an insulating state characterized by a linear l-SDW plus a small CDW with $\phi_{\rho}=0$, accompanied by a magnetization $m^{z}=1 / 3$ (phase A'). This collinear state lies above the s-SDW by only a small energy difference (of order $0.03 t_{1}$ per site), and could be stabilized by other factors (e.g., spin-orbit). A recent LSDA calculation for $\sqrt{3}$ - $\mathrm{Si} / \mathrm{Si}(111)$ has indicated this l-SDW as the ground state, at least if spins are forced to be collinear 15 The HF bands for this solution are shown in Fig. 5(b), and are very similar to the LSDA surface band for $\mathrm{Si} / \mathrm{Si}(111)$. The phase $\phi_{\rho}=0$ of the CDW order parameter corresponds to a real-space charge distribution in which one sublattice has a charge $1+2\left|\rho_{\mathbf{K}}\right|$, while the remaining two are equivalent and have charges $1-\left|\rho_{\mathbf{K}}\right|$, compatible with the experimental findings on $\mathrm{Sn} / \mathrm{Ge}(111)$ and $\mathrm{Pb} / \mathrm{Ge}(111)$. The amplitude $\left|\rho_{\mathbf{K}}\right|$ of the $\mathrm{CDW}$ is in general quite small in this phase. It should be noted, however, that a STM map is not simply a direct measure of the total charge density.2425 This will be discussed in sect. $\mathrm{V}$.

Phase B': Asymmetric CDW with $m^{z}=1 / 3$ insulating phase. By increasing the n.n. repulsion $V$, the energies of the s-SDW and of the l-SDW tend to approach, until they cross at a critical value $V_{c}$ of $V$. At $U / t_{1}=10$ we find $V_{c} / t_{1} \approx 3.3$ for model A, $V_{c} / t_{1} \approx 6.6$ for model B. As $V>V_{c}$, however, an insulating asymmetric CDW (a-CDW) prevails. This is simply the spin collinear version of the non-collinear phase described in Sect. [II B. Fig. 6 shows the energy per site of the most relevant HF solutions at $U / t_{1}=10$ as a function of $V$ for model B (Coulomb tail case). The s-SDW and the l-SDW cross at $V_{c} \approx 6.6 t_{1}$ where, however, the a-CDW insulating solution starts to be the favored one. This large- $V$ solution has a large $C D W$ order parameter with $\phi_{\rho} \neq 0(\bmod .2 \pi / 3)$, a concomitant l-SDW, and $m^{z}=1 / 3$. By recalling the discussion in sect. III B, we notice that a state with a magnetization $m^{z}=1 / 3$ and a l-SDW is the best HF solution once a $3 \times 3$ restriction has been applied, since a spiral SDW on the singly occupied sublattice would involve a larger periodicity (phase B).

Phase D: Symmetric non-magnetic CDW metallic phase. For small values of $U$ and $V$, or for large enough e-ph coupling $g$, a metallic CDW with $\phi_{\rho}=0$ (m-CDW) is found. (See Fig. F(c) for the HF bands.) This phase 
constitutes a candidate, alternative to the magnetic phase B', and compatible with the main experimental facts, which might be relevant for the case of $\mathrm{Pb} / \mathrm{Ge}(111)$ and of $\mathrm{Sn} / \mathrm{Ge}(111)$. The degree of metallicity of this phase is much reduced relative to the undistorted surface (pseudo-gap).

We stress that the e-ph interaction can stabilize the $\phi_{\rho}=0 \mathrm{~m}$-CDW also at relatively large $U$, by countering $U$ with a large negative $\Delta U=-g^{2} / M \omega_{\mathrm{K}}^{2}$. We demonstrate this in Fig. 7, where we plot the energy per site as a function of $\Delta U$ at $U / t_{1}=8$ and $V / t_{1}=2$, for the three relevant HF solutions, i.e., the spiral SDW (phase A), the collinear SDW with $m^{z}=1 / 3$ (phase A'), and the metallic non-magnetic CDW (phase D). The spiral SDW is unaffected by the electron-phonon coupling. The energy of the collinear SDW with $m^{z}=1 / 3$ improves a little bit by increasing $g$, due to the small CDW amplitude of this phase. This effect is not large enough as to make this phase stable in any range of couplings. At a critical value of $g$, the metallic non-magnetic CDW (where the CDW order parameter is large, $\left.\left|\rho_{\mathbf{K}}\right| \sim 0.5\right)$ wins over the magnetic phases. The Fourier transform of the lattice distortion at $\mathbf{K}$ is given by $\left\langle z_{\mathbf{K}}\right\rangle=\left(g / M \omega_{\mathbf{K}}^{2}\right) \rho_{\mathbf{K}}=\rho_{b f K}|\Delta U| / g$.

A rough estimate shows that the order of magnitude of the electron-phonon coupling necessary to stabilize the CDW phase is not unreasonable. With $g=1 \mathrm{eV} / \AA A, M_{S i}=28$, and $\omega_{\mathrm{K}} \approx 30 \mathrm{meV}$ we get $\Delta U \approx-3 t_{1}$, sufficient to switch from a s-SDW ground state to a m-CDW for $U / t_{1}=8$ and $V / t_{1}=2$. With these values of the parameters we have $\left|\rho_{\mathbf{K}}\right| \approx 0.43$, and we estimate $\left|\left\langle z_{\mathbf{K}}\right\rangle\right| \approx 0.07 \AA$. This corresponds, since $\left\langle z_{\mathbf{r}}\right\rangle \sim 2 \cos (\mathbf{K} \cdot \mathbf{r})\left|\left\langle z_{\mathbf{K}}\right\rangle\right|$, to a total displacement between the adatom going up and the two going down of $\Delta z=3\left|\left\langle z_{\mathbf{K}}\right\rangle\right| \approx 0.2 \AA$.

We notice that values of $g$ much larger than those used in Fig. 7 would eventually stabilize a superconducting ground state (see Appendix).

\section{CDW ORDER PARAMETER AND STM EXPERIMENTS}

We discuss, in the present section, the relationship between the CDW order parameter, as defined in Eq. 9, and an STM map of the surface. As the crudest approximation to the tunneling current for a given bias $V_{\text {bias }}$ we consider the integral of the charge density for one-electron states within $V_{\text {bias }}$ from the Fermi level, weighted with barrier tunneling factor $T(V) 2,25$

$$
J\left(V_{\text {bias }}, \mathbf{r}=x, y ; z\right) \approx \int_{0}^{V_{\text {bias }}} d V \sum_{n \mathbf{k}}\left|\Psi_{n \mathbf{k}}(\mathbf{r})\right|^{2} \delta\left(E_{n \mathbf{k}}-E_{F}+V\right) T(V) .
$$

The tunneling factor leads to weighting prominently the states immediately close to the Fermi level. In view of the purely qualitative value of Eq. (15), we have moreover decided to ignore $T(V)$ altogether and to account for its effect by reducing the bias voltage $V_{\text {eff }}$ in Eq. (15), to an effective value $V_{\text {bias }}^{\text {eff }}$. By doing this, we have extracted an "STM map" for a point in phase A' $\left(U / t_{1}=9\right.$ and $V=2$, model A) - a spin-density waves where the amplitude of the CDW order parameter is rather small, $\left|\rho_{\mathbf{K}}\right|=0.039$ - and a point in phase $\mathrm{D}\left(U / t_{1}=4\right.$ and $V=2$, model A) - a pure CDW where the order parameter is quite large, $\left|\rho_{\mathbf{K}}\right|=0.4$. The results for constant $z$, and $x, y$ moving from adatom A to $\mathrm{B}$ to C, are shown in Fig. B(a) and (b), for the two cases. The solid curves refer to positive bias (current flowing from the sample to the tip), probing occupied states close to the Fermi level. The dashed curve refers to negative bias, probing unoccupied states. In both cases a) and b), one of the three atoms yields a larger current at positive bias, while the other two atoms have larger currents at negative bias. The insets show the predicted "contrast" between the two peak values, $\left(J_{1}-J_{2}\right) /\left(J_{1}+J_{2}\right), J_{1}$ and $J_{2}$ being in each case, respectively, the largest and the smallest of the STM peak currents at the positions of the adatoms. We notice the following points: i) for the occupied states (positive bias) the pure CDW phase has, as expected, a larger contrast than the magnetic phase. As we neglect the tunneling factors $T(V)$, in the limit of large positive effective bias we recover the total asymmetry in the charge of the two inequivalent atoms, $\left(n_{1}-n_{2}\right) /\left(n_{1}+n_{2}\right)$, indicated by a dashed horizontal line in the insets. Observe that the way this large bias limit is reached is completely different for the two cases a) and b): in the magnetic case a) the contrast overshoots at small biases attaining values substantially larger than the nominal CDW order parameter, and then goes to the limit $\left(n_{1}-n_{2}\right) /\left(n_{1}+n_{2}\right)$ from above; in the pure CDW case b), on the contrary, the limit is reached monotonically from below. ii) for empty states (negative bias) the contrast is even more surprising: at small bias it is very large in both cases a) and b). By increasing the bias, the contrast for the pure CDW case tends monotonically to a large value, whereas the magnetic case shows a strong non monotonicity.

These results suggest that one should look more carefully, and quantitatively, at the behavior of the asymmetry between STM peak currents as a function of the bias, including the region of relatively small biases: the different behavior of the asymmetry of the magnetic case versus the pure CDW case should be marked enough - and survive in a more refined analysis including $T(V)$ - as to make the STM map a good way of discriminating between the two scenarios. 


\section{DISCUSSION AND CONCLUSIONS}

Within our model study we have learned that on the surfaces considered:

(i) If $U$ and $V$ are ignored, there is no straight electron-phonon driven $3 \times 3$ CDW surface instability. However, any phase involving a CDW, for example as a secondary order parameter attached to a primary SDW, can take advantage and gain some extra stabilization energy from a small surface lattice distortion, via electron-phonon coupling.

(ii) Electron-electron repulsion and the two-dimensional Fermi Surface are capable of driving transitions of the undistorted metallic surface to a variety of states, that are either insulating or in any case less metallic, some possessing the $3 \times 3$ periodicity.

(iii) This can occur via two different mechanisms: a) the inter-site repulsion $V$ can stabilize insulating or semimetallic CDWs, without a crucial involvement of spin degrees of freedom; b) the on-site repulsion $U$ can produce essentially magnetic insulators with or without a weak accompanying $3 \times 3 \mathrm{CDW}$, as required by symmetry.

(iv) For $U$ moderate of order $W$ and for smaller $V$, an interesting state is realized, with a large SDW and a small accompanying CDW. The state is either a small-gap insulator, or a semi-metal, and may or may not be associated with a net overall magnetization, depending on the nature (linear or spiral, respectively) of the leading SDW.

(v) For $U$ and $V$ both small but finite, a metallic CDW without any magnetism is obtained. The same phase can also be stabilized for larger values of $U$ by the presence of a substantial electron-phonon coupling. We stress that, in this case, $V$ is the coupling responsible for the $3 \times 3$ symmetry of the unit cell, whereas the role of the electron-phonon coupling is that of destroying magnetism by effectively decreasing $U$. Electron-phonon coupling alone is not sufficient to justify a commensurate $3 \times 3 \mathrm{CDW}$.

(vi) Either of the phases in (iv) or (v) could be natural candidates for explaining the weak $3 \times 3$ CDW seen experimentally on $\mathrm{Sn}-\mathrm{Pb} / \mathrm{Ge}(111)$.

(vii) Finally, for large $U$, small $V$ (in comparison with the bandwidth $W$ ) the Mott-Hubbard state prevails. It is a wide-gap insulator, with a pure spiral SDW, with $3 \times 3$ overall periodicity, and coplanar $120^{\circ}$ long-range spin ordering at zero temperature. It possesses no net magnetization, and no accompanying CDW.

(viii) The above is the kind of state which we expect to be realized on $\mathrm{SiC}(0001)$, and also possibly on $\mathrm{K} / \mathrm{Si}(111)$ :B.

Among existing experiments, we have addressed particularly photoemission and STM. Our calculated band structure for both the SDW/CDW state A' (iv) and the pure CDW state D (v) exhibit features which are similar to those found in photoemission from $\mathrm{Sn}-\mathrm{Pb} / \mathrm{Ge}(111) \mathrm{B}$ 国 The simulated STM images for the two kind of states are predicted to differ in their voltage dependence.

Future experiments are strongly called for, aimed at detecting whether magnetic correlations are actually dominant, as we think is very likely, on all these surfaces, or whether $\mathrm{Sn}-\mathrm{Pb} / \mathrm{Ge}(111)$ are instead non-magnetic and electronphonon driven. The issue of whether magnetic long-range order - which we definitely propose for $\operatorname{SiC}(0001)$ and $\mathrm{K} / \mathrm{Si}(111)$ :B at $T=0$, and also hypothesize for $\mathrm{Sn}-\mathrm{Pb} / \mathrm{Ge}(111)$ - survives up to finite temperatures is one which we cannot settle at this moment. This due to the difficulty in estimating the surface magnetic anisotropy, without which order would of course be washed out by temperature. In any case, it should be possible to pursue the possibility of either magnetism or incipient magnetism using the appropriate spectroscopic tools.

This line of experimental research, although undoubtedly difficult, should be very exciting since it might lead to the unprecedented discovery of magnetic states at surfaces possessing no transition metal ions of any kind, such as these seemingly innocent semiconductor surfaces.

We acknowledge financial support from INFM, through projects LOTUS and HTSC, and from EU, through ERBCHRXCT940438. We thank S. Modesti, J. Lorenzana, M.C. Asensio, J. Avila, G. Le Lay, E.W. Plummer and his collaborators, for discussions.

\section{APPENDIX. LARGE NEGATIVE $U$ : A SUPERCONDUCTING GROUND STATE.}

The limit of large negative $U, U \rightarrow-\infty$, is considered here to show that CDWs are not favored by on-site attraction alone. Instead, a superconducting ground state is favored.16 To see this, consider the real-space states which are the low energy configurations for $U \rightarrow-\infty$ : they consist of $N_{e} / 2$ sites (if $N_{e}$ is the number of electrons) each of which is occupied by a pair of electrons with opposite spins. The large degeneracy in this manifold of states is - once again, like in the $U \rightarrow \infty$ case - removed by kinetic energy in second order perturbation theory. By assigning a pseudo-spin- $1 / 2$ state to each site (up, if occupied by a pair, down if empty) one can show that the effective Hamiltonian is 16

$$
H_{\mathrm{eff}}=-\sum_{(i j)} \frac{J_{i j}^{\perp}}{2}\left(S_{\mathbf{r}_{i}}^{+} S_{\mathbf{r}_{j}}^{-}+\text {H.c. }\right)+\sum_{(i j)} J_{i j}^{z} S_{\mathbf{r}_{i}}^{z} S_{\mathbf{r}_{j}}^{z},
$$


with $J_{i j}^{\perp}=4\left|t_{i j}\right|^{2} /|U|$ and $J_{i j}^{z}=J_{i j}^{\perp}$. If $V$-terms are added, $J^{z}$ is modified to $J_{i j}^{z}=J_{i j}^{\perp}+4 V_{i j}$. Restricting our consideration to the n.n. case, we are left with a n.n. Heisenberg Hamiltonian with ferromagnetic xy-part and an antiferromagnetic z-part. The sign of the xy-part cannot be changed at will by a canonical transformation because the lattice is non-bipartite. The result is that the order is in the plane (i.e., superconductivity wins) for small $V$. Only if $V$ is large enough the CDW (i.e., order in the z-direction) will be favored.

Entirely similar considerations apply to the case of strong electron-phonon coupling, $g \rightarrow \infty$.

${ }^{1}$ J. M. Carpinelli et al., Nature 381, 398 (1996).

${ }^{2}$ A. Goldoni, C. Cepek, S. Modesti, Phys. Rev. B 55, 4109 (1997).

${ }^{3}$ A. Goldoni and S. Modesti, Phys. Rev. Lett. 79, 3266 (1997); S. Modesti (private commun.)

${ }^{4}$ J. Avila, A. Mascaraque, E.G. Michel, and M.C. Asensio, Appl. Surf. Sci. 123/124, 626 (1998).

${ }^{5}$ G. Le Lay et al., Appl. Surf. Sci. 123/124, 440 (1998).

${ }^{6}$ J. M. Carpinelli, H. H. Weitering, M. Bartkowiak, R. Stumpf, and E. W. Plummer, Phys. Rev. Lett. 79, 2859 (1997) .

${ }^{7}$ A. Mascaraque, J. Avila, E.G. Michel, and M.C. Asensio, Phys. Rev. B (to be published).

${ }^{8}$ H. H. Weitering et al., Phys. Rev. Lett. 78, 1331 (1997).

${ }^{9}$ L. I. Johansson et al., Surf. Sci. 360, L478 (1996); J.-M. Themlin et al., Europhys. Lett. 39, 61 (1997).

${ }^{10}$ G. Santoro, S. Sorella, F. Becca, S. Scandolo and E. Tosatti, Surf. Sci. 402-404, 802 (1998).

11 J.E. Northrup and J. Neugebauer, Phys. Rev. B 57, R4230 (1998).

${ }^{12}$ V. Anisimov, G. Santoro, S. Scandolo, and E. Tosatti (work in progress).

${ }^{13}$ E. Tosatti and P. W. Anderson, in Proc. 2nd Int. Conf. on Solid Surfaces, ed. S. Kawaji, Jap. J. Appl. Phys., Pt. 2, Suppl. 2, 381 (1974); E. Tosatti, in Electronic surface and interface states on metallic systems, p. 67, eds. E. Bertel and M. Donath (World Scientific, Singapore, 1995).

${ }^{14} \mathrm{~S}$. Scandolo et al., to be published.

${ }^{15}$ S. Scandolo, F. Ancilotto, G. L. Chiarotti, G. Santoro, S. Serra, and E. Tosatti, Surf. Sci. 402-404, 808 (1998).

${ }^{16}$ R. R. dos Santos, Phys. Rev. B 48, 3976 (1993).

${ }^{17}$ We observe that, in principle, non-diagonal terms, i.e., terms which cannot be recast in the form of a density-density interaction, should be included. However, the magnitude of such terms - which involve overlap integrals between Wannier orbitals at different adatoms - can be estimated to be quite smaller than the diagonal terms we keep.

18 M. Sabisch, P. Kruger, and J. Pollmann, Phys. Rev. B 55, 10561 (1997).

${ }^{19}$ P. W. Anderson in Frontiers and Borderlines in Many-Particle Physics, Proc. E. Fermi Summer School in Varenna, july 1987 (North-Holland, Amsterdam, 1988).

${ }^{20}$ G.D. Mahan, Many-Particle Physics, 2nd ed. (Plenum Press, New York, 1990).

${ }^{21}$ H. R. Krishnamurthy et al., Phys. Rev. Lett. 64, 950 (1990); C. Jayaprakash et al., Europhys. Lett. 15, 625 (1991).

${ }^{22}$ See J. Tolédano and P. Tolédano, The Landau theory of phase transitions, (World Scientific, Singapore, 1987).

${ }^{23}$ O. Zachar, S. A. Kivelson, and V. J. Emery, (preprint)

24 A. Selloni, P. Carnevali, E. Tosatti and C. D. Chen, Phys. Rev. B 31, 2602 (1985).

${ }^{25}$ E. Tosatti, in Highlights in Condensed Matter Physics and Future Prospects, p. 631, ed. L. Esaki, Plenum Press (New York, 1991). 


\section{Figure Captions}

FIG. 1. Surface state dispersion for hypothetical $\mathrm{Si} / \mathrm{Si}(111)$, as obtained from gradient corrected LDA (solid squares). A very similar band is obtained for $\mathrm{Pb} / \mathrm{Ge}(111)$ and $\mathrm{Sn} / \mathrm{Ge}(111)$. The solid line is a tight-binding fit obtained by including up to the sixth shell of neighbors, $t_{1}, \cdots, t_{6}$. The fit gives $t_{1}=0.0551 \mathrm{eV}$, and $t_{2} / t_{1}=-0.3494, t_{3} / t_{1}=0.1335, t_{4} / t_{1}=-0.0615$, $t_{5} / t_{1}=0.0042, t_{6} / t_{1}=-0.0215$. The dashed line is the best fit using $t_{1}$ and $t_{2}$ only. Upper inset: The Fermi surface of the half-filled surface band. The outer hexagon is the BZ of the $\sqrt{3} \times \sqrt{3}$ phase, and the inner hexagon is the BZ of the $3 \times 3$ phase. Notice the poor nesting at the BZ corner wavevector $\mathbf{K}=(4 \pi / 3 a, 0)$, joining two opposite $M_{3 \times 3}$ points. Lower inset: The zero temperature Lindhard response function $\chi_{o}(\mathbf{q})$ for the half-filled surface band. Notice the two peaks located at $\mathbf{q}_{1} \approx 0.525 \mathbf{K}$ and $\mathbf{q}_{2} \approx 1.32 \mathbf{K}$, and no feature whatsoever at $\mathbf{K}$, indicating poor nesting.

FIG. 2. Density contours of the Wannier function associated with the $\mathrm{Si} / \mathrm{Si}(111)$ surface band, calculated with gradient-corrected LDA: dots correspond to atomic positions.

FIG. 3. The CDW on the adatom triangular lattice in the limit $t_{i j}=0, U \ll V$. Sublattice A is doubly occupied, sublattice $\mathrm{B}$ is singly occupied, and $\mathrm{C}$ is empty. The large spin degeneracy associated to the unpaired singly occupied sites on sublattice $\mathrm{B}$ is removed by the next-nearest neighbor hopping $t_{2}$ in second order perturbation theory. The arrow indicates a possible virtual process, leading to an extra doubly occupied site (with an associated energy gap of $U$ ), which generates the standard antiferromagnetic exchange coupling between the spins on sublattice B.

FIG. 4. Schematic Hartree-Fock phase diagram of model A (nearest neighbor $V$ only, $g=0$ ) for the band structure shown in Fig. 1. Only the most important commensurate $3 \times 3$ phases have been studied. (Details of the merging of the various lines are not accurate.) Left figure: phase obtained allowing spin non-collinearity. Right figure: strictly collinear phase diagram. The non-vanishing order parameters of the different phases are as follows: $\mathrm{A}: \mathbf{S}_{\mathbf{K}}=\left|\mathbf{S}_{\mathbf{K}}\right| / \sqrt{2}(1,-i, 0) ; \mathrm{A}^{\prime}: \mathbf{S}_{\mathbf{K}}=\left|\mathbf{S}_{\mathbf{K}}\right|(0,0,1)$, $m_{z}=1 / 3, \rho_{\mathbf{K}}=\left|\rho_{\mathbf{K}}\right| ; \mathbf{B}^{\prime}: \mathbf{S}_{\mathbf{K}}=\left|\mathbf{S}_{\mathbf{K}}\right|(0,0,1), m_{z}=1 / 3, \rho_{\mathbf{K}}=\left|\rho_{\mathbf{K}}\right| e^{i \phi_{\rho}}$ (with $\left.0<\phi_{\rho}<\pi / 6\right)$; B: $3 \sqrt{3}$ extension of phase B' when allowing for non-collinearity of the unpaired spins. C and C' are semi-metallic versions of A and A', respectively. $\mathrm{D}: \rho_{\mathbf{K}}=\left|\rho_{\mathbf{K}}\right| ; \mathrm{E}: \mathbf{S}_{\mathbf{K}}=i\left|\mathbf{S}_{\mathbf{K}}\right|(0,0,1), \rho_{\mathbf{K}}=-\left|\rho_{\mathbf{K}}\right| ; \mathrm{F}: \rho_{\mathbf{K}}=\left|\rho_{\mathbf{K}}\right| e^{i \phi_{\rho}}$ (with $0<\phi_{\rho}<\pi / 6$ ); IM: Incommensurate metallic SDW/CDW; PM: Paramagnetic metal. Phases A(A'), and B(B') are insulating and magnetic. Phases $\mathrm{C}\left(\mathrm{C}^{\prime}\right)$, E, and IM are metallic and magnetic. Phase D is a pure CDW and is metallic. Phases A', C', and D have CDW order parameter with the same symmetry as observed on $\mathrm{Pb} / \mathrm{Ge}(111)$ and $\mathrm{Sn} / \mathrm{Ge}(111)$. The effect of a finite electron-phonon coupling $(g \neq 0)$ is discussed in the text.

FIG. 5. Plot of the HF electronic bands along high symmetry directions of the BZ for the s-SDW and two CDW $\phi_{\rho}=0$ solutions: (a) at $U / t_{1}=9$ and $V / t_{1}=2$, the insulating s-SDW (phase A, ground state); (b) at $U / t_{1}=9$ and $V / t_{1}=2$, the insulating solution with a small CDW and $m^{z}=1 / 3$ (phase A', meta-stable, the actual ground state being the s-SDW); Solid and dashed lines denote up and down bands, respectively. (c) at $U / t_{1}=4$ and $V / t_{1}=2$, the metallic solution with a large CDW and no magnetism (phase D). The band structure for phases C and C' are similar to A and A' except for band overlap, making them semi-metallic. Insets indicate the charge and spin imbalance (when present) between the three adatoms in the $3 \times 3$ unit cell.

FIG. 6. Energy per particle, as a function of $V$ at $U / t_{1}=10$, for the commensurate HF solutions of phase A, A', and B', obtained for model B (Coulomb tail case). The results for model A are entirely similar, with $V_{c} \approx 3.3 t_{1}$.

FIG. 7. The energy per site, as a function of the electron-phonon coupling $-\Delta U=g^{2} / M \omega_{\mathbf{K}}^{2}$, at $U=8 t_{1}$ and $V=2 t_{1}$ for the model with n.n. interactions only, for the three relevant HF solutions, i.e., the spiral SDW, the collinear SDW with $m^{z}=1 / 3$, and the metallic non-magnetic CDW.

FIG. 8. (a) STM map for tip motion (at constant height) along the triangle indicated, for the l-SDW state at $U / t_{1}=9$, $V=2$. The result is obtained from Eq. 15 without explicitly including $T(V)$, but reducing the bias to an effective one, $V_{\text {bias }}^{\text {eff }}=6 t_{1}$, thus imposing that only states sufficiently close to the Fermi level contribute significantly to the STM current. The solid and dashed curves refer, respectively, to positive and negative STM bias. The inset shows the "contrast" between the peaks (see text) as a function of the effective bias $V_{\text {bias. }}^{\text {eff }}$ (b) Same as (a) for a pure CDW state in phase D. 


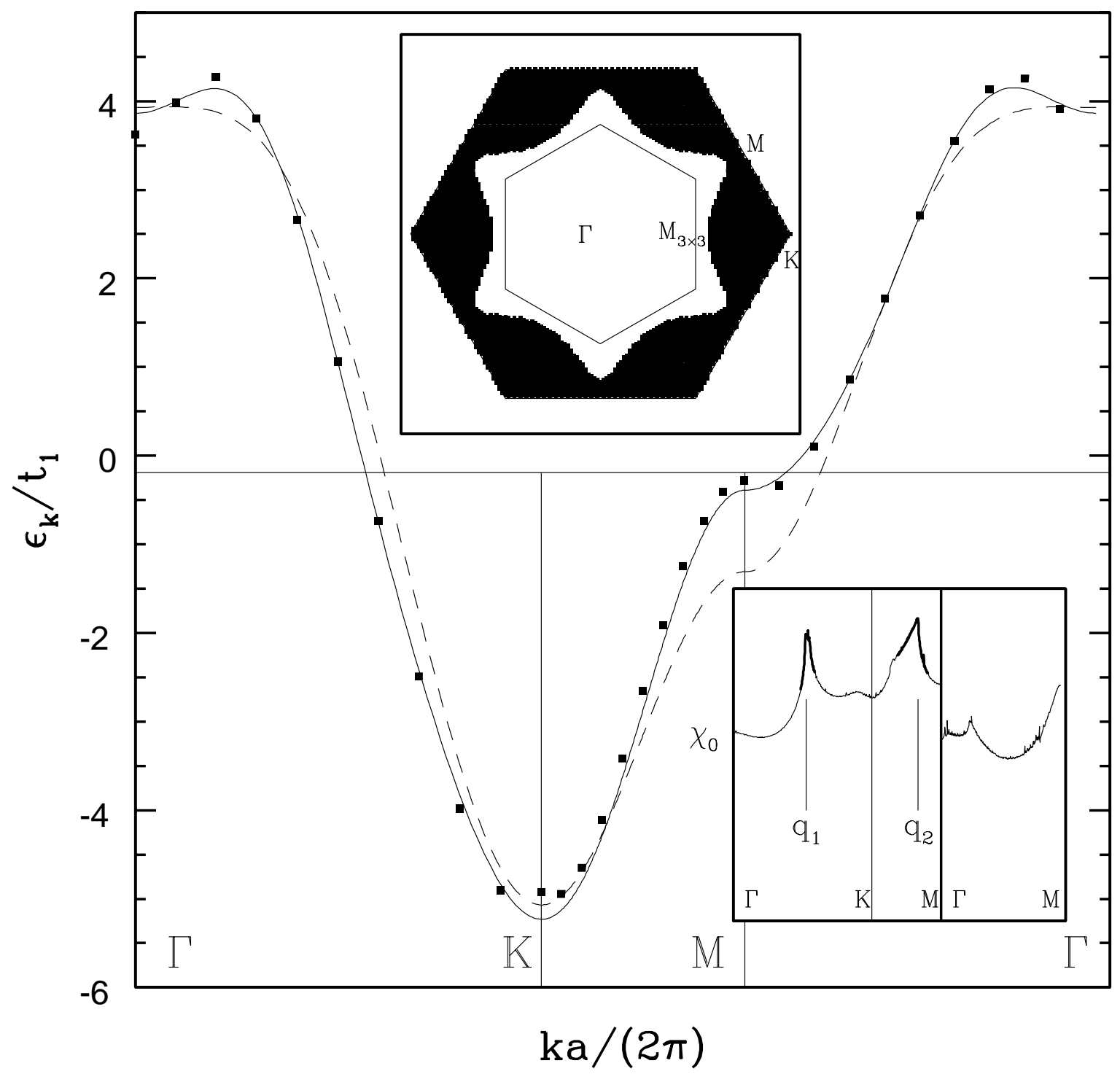




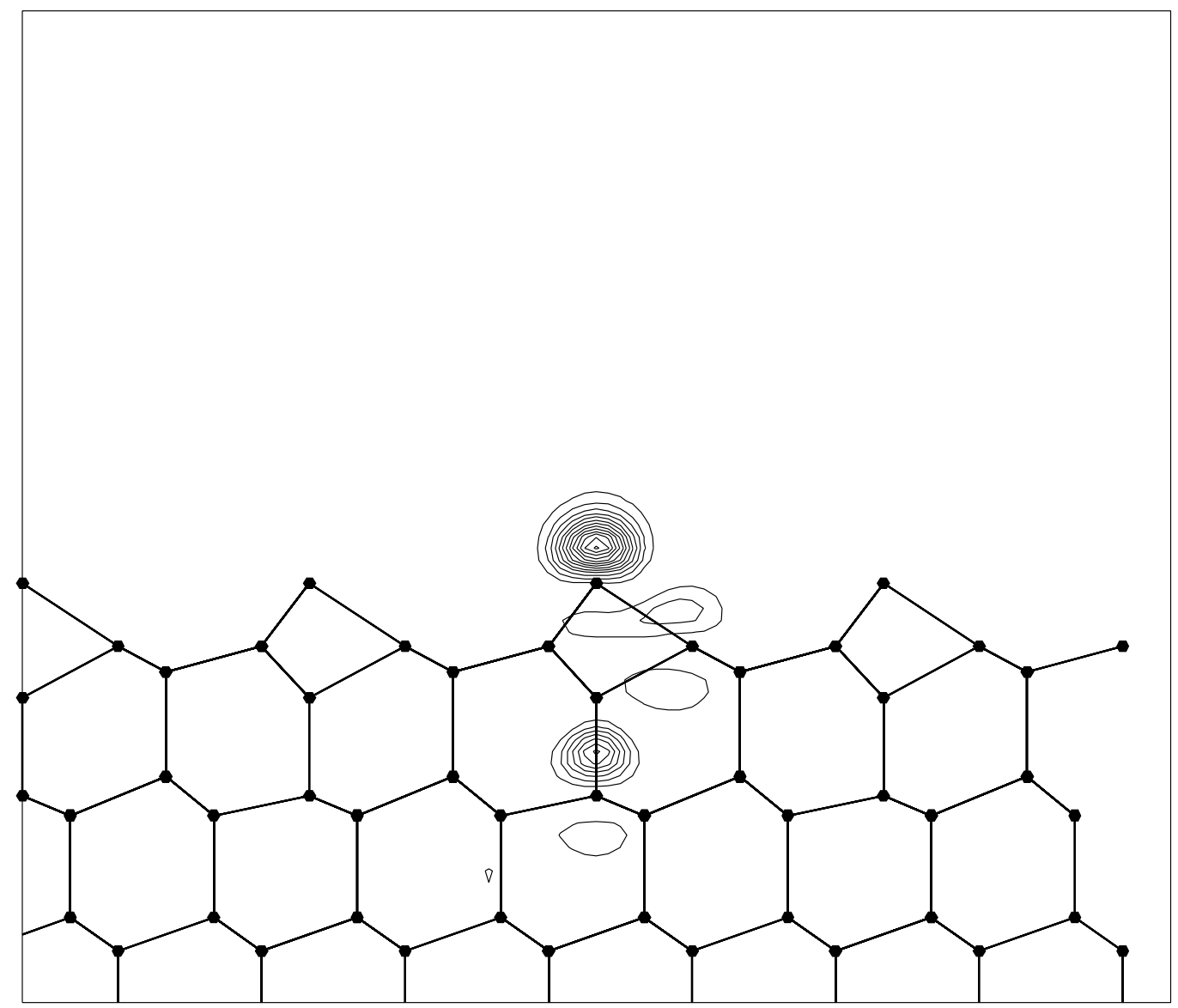




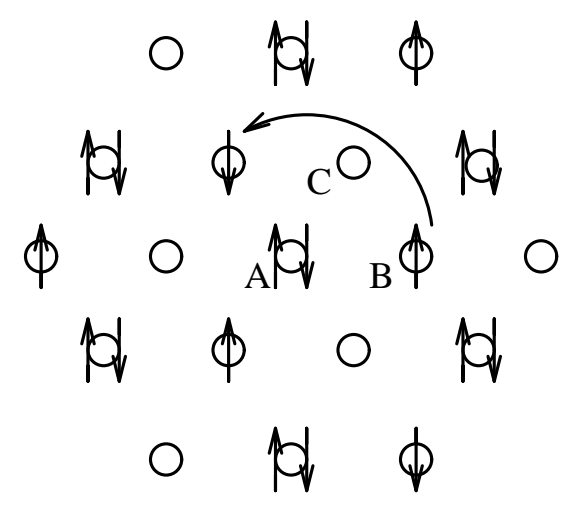




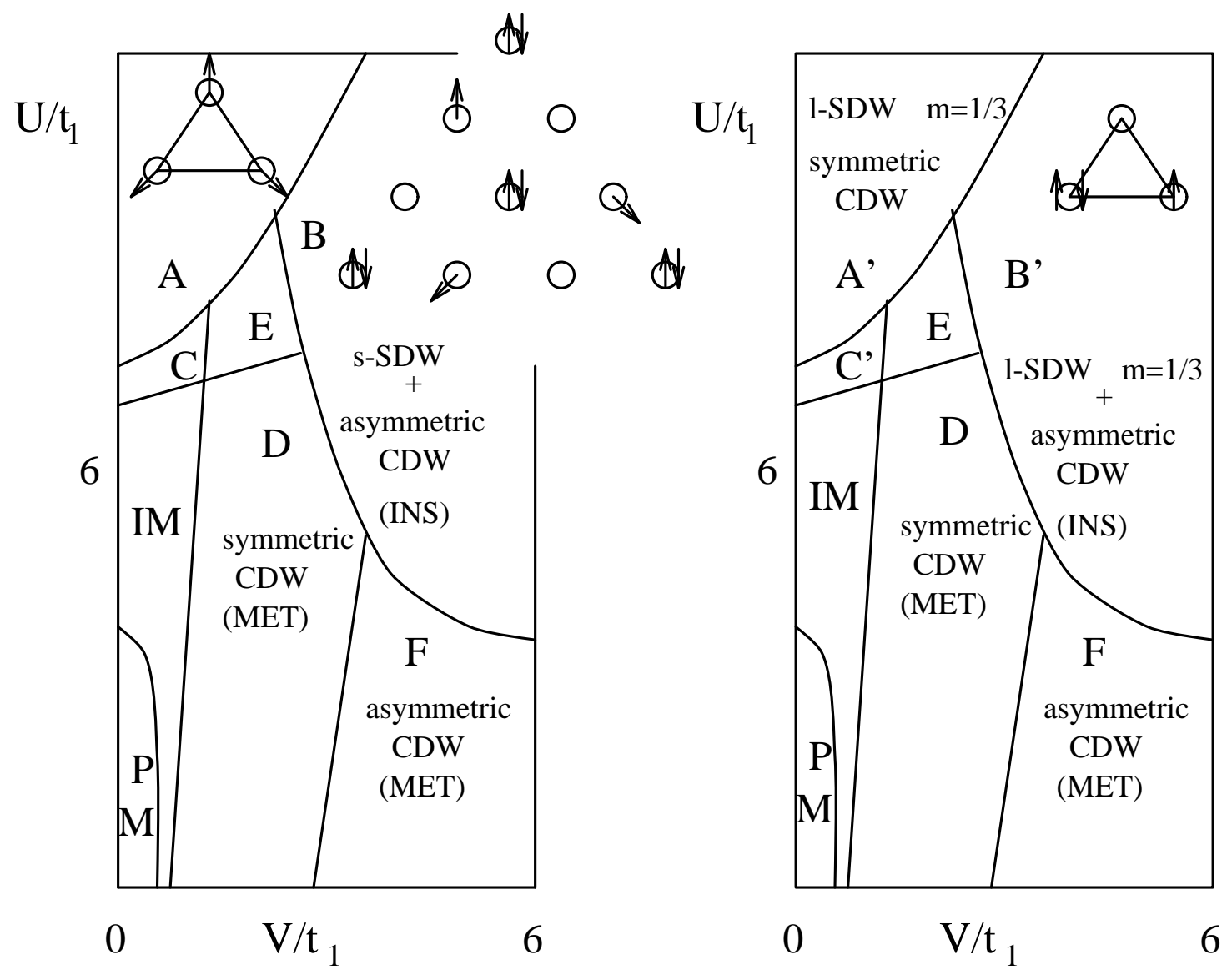




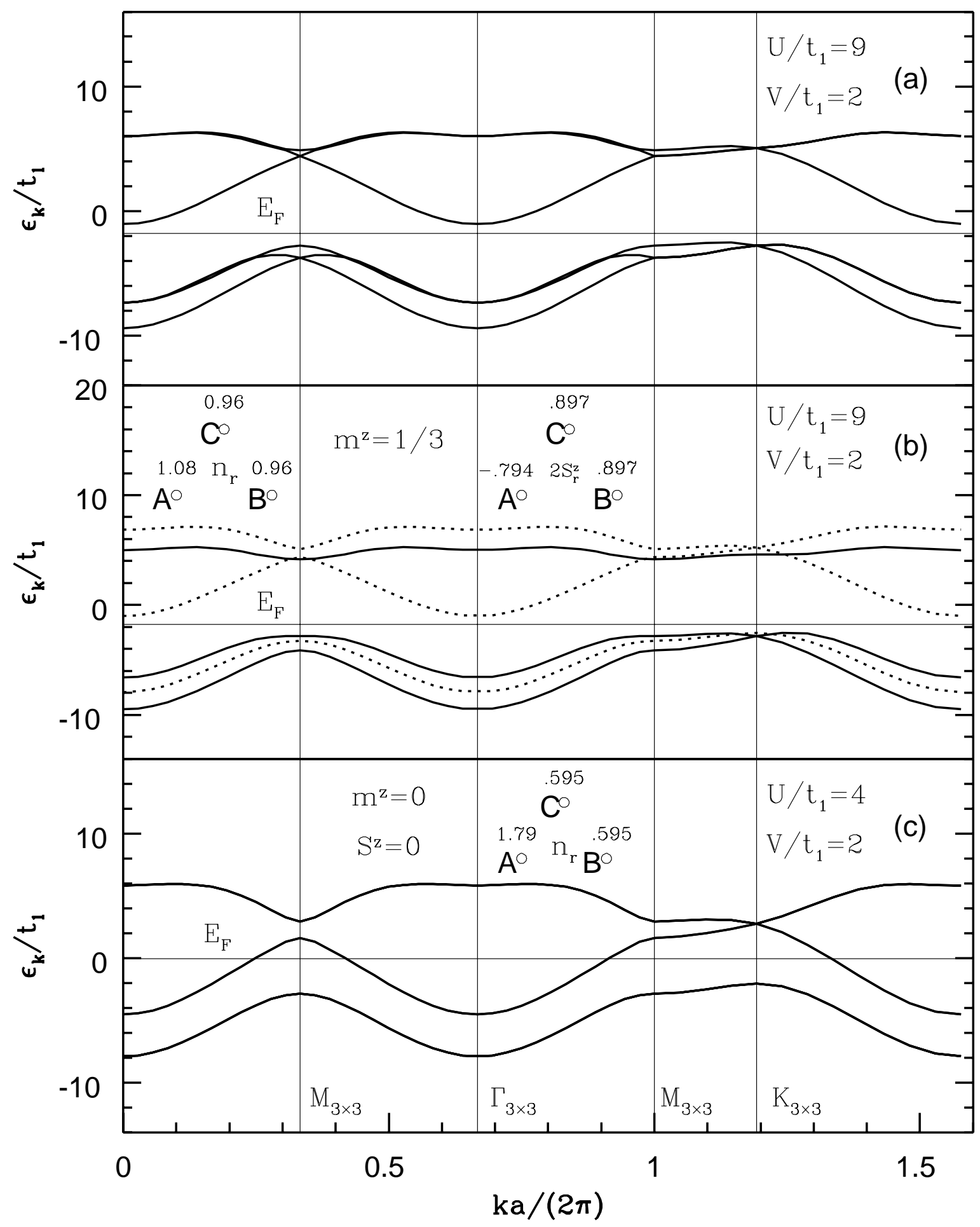




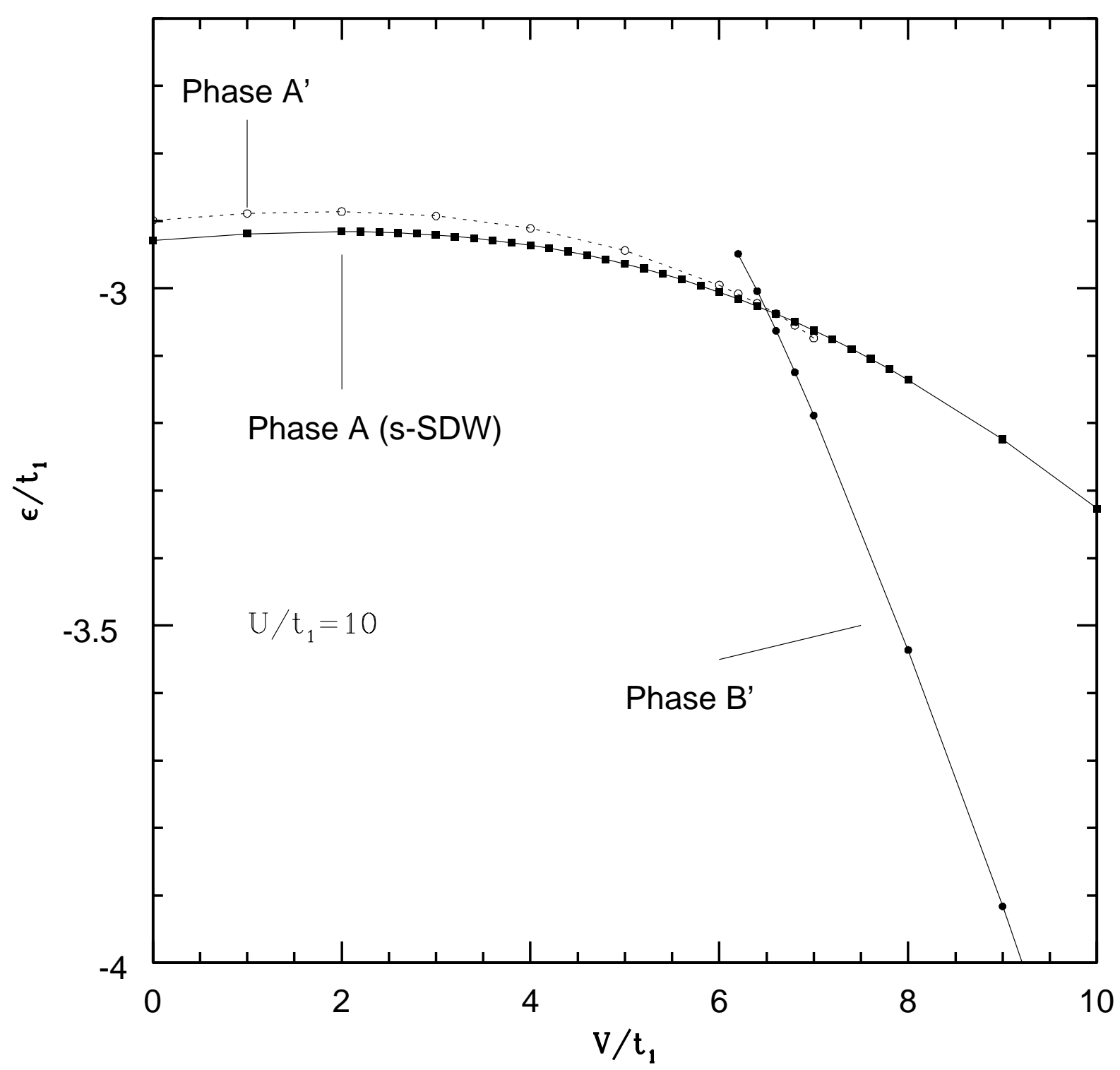




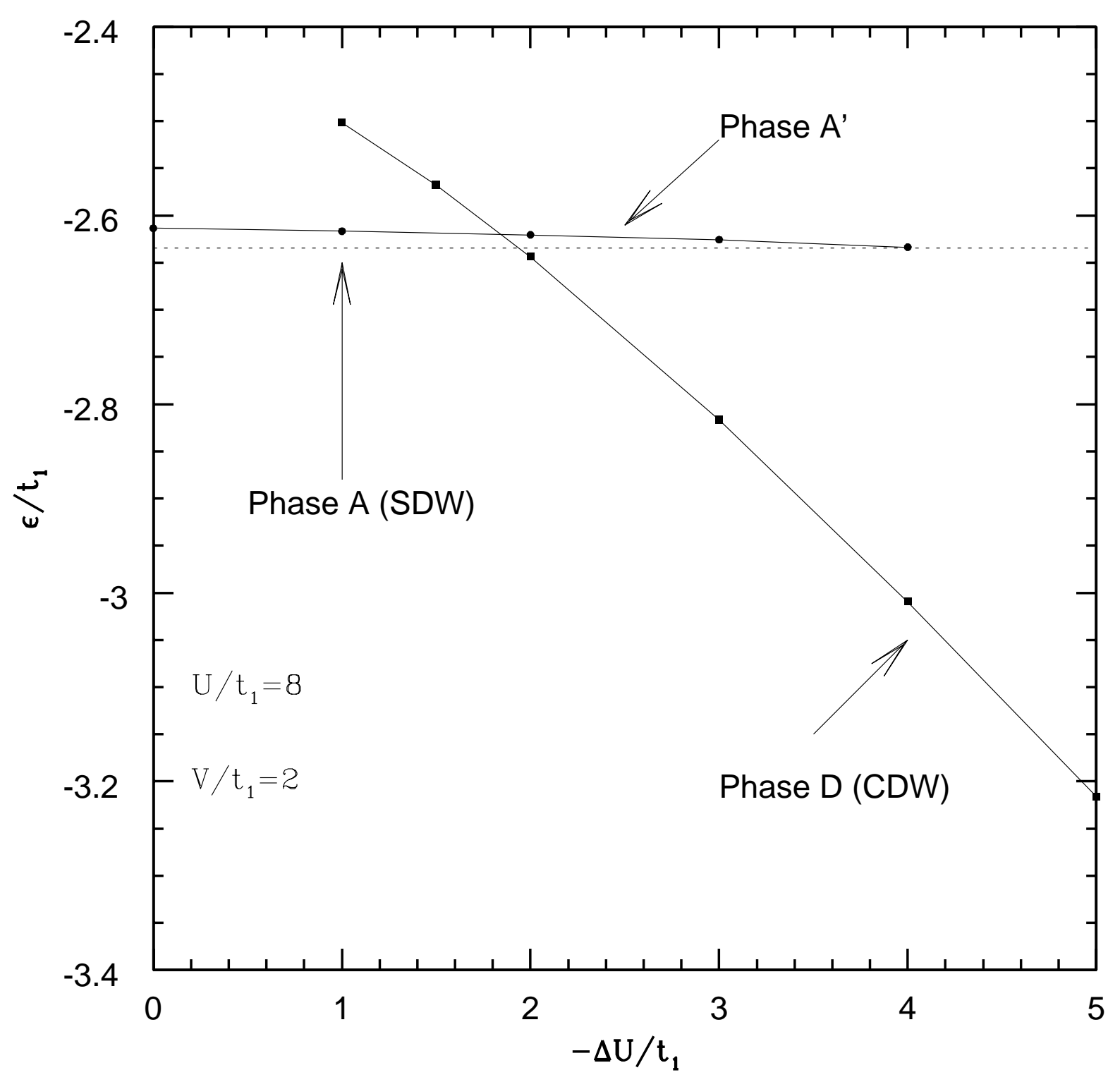




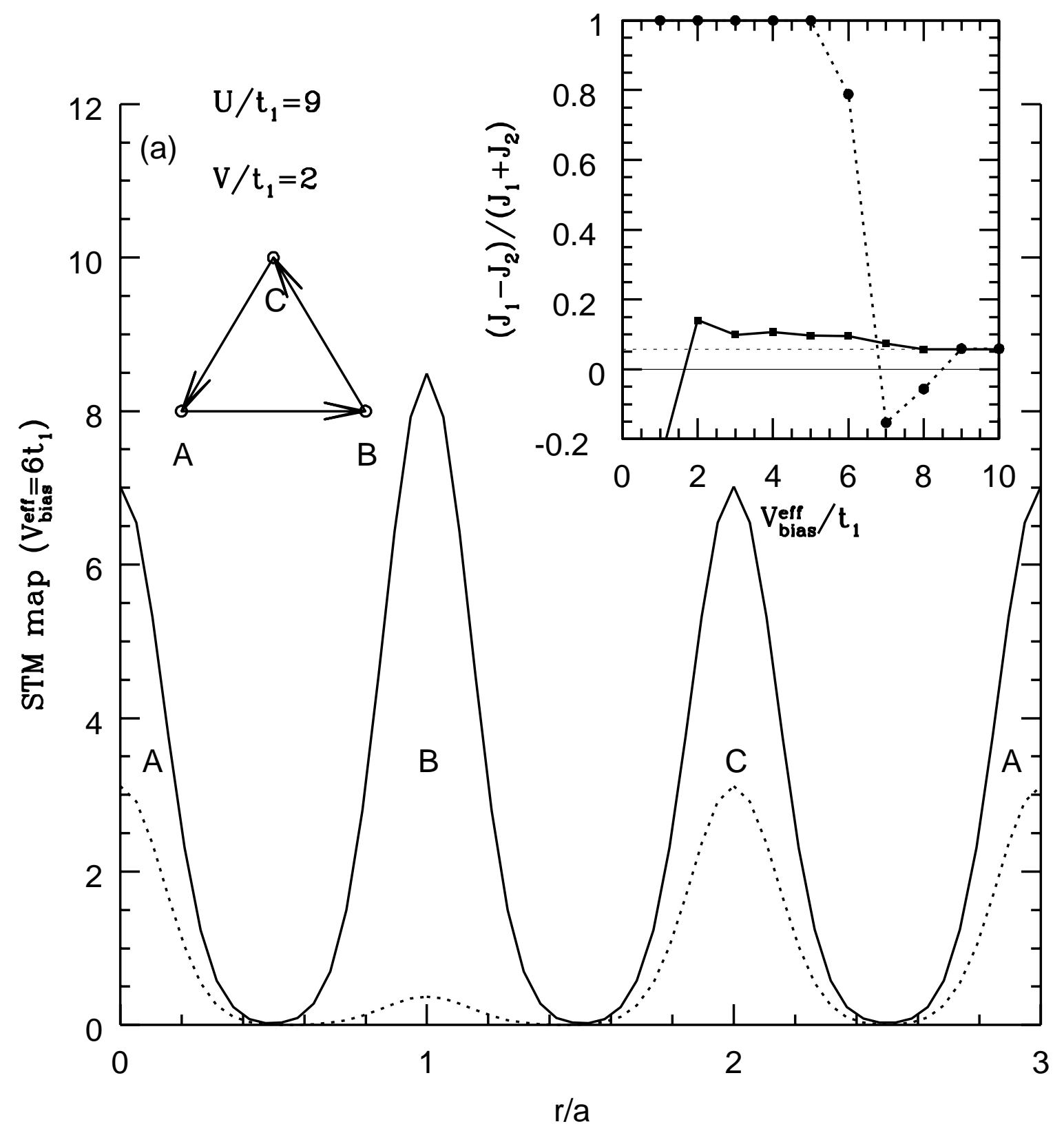




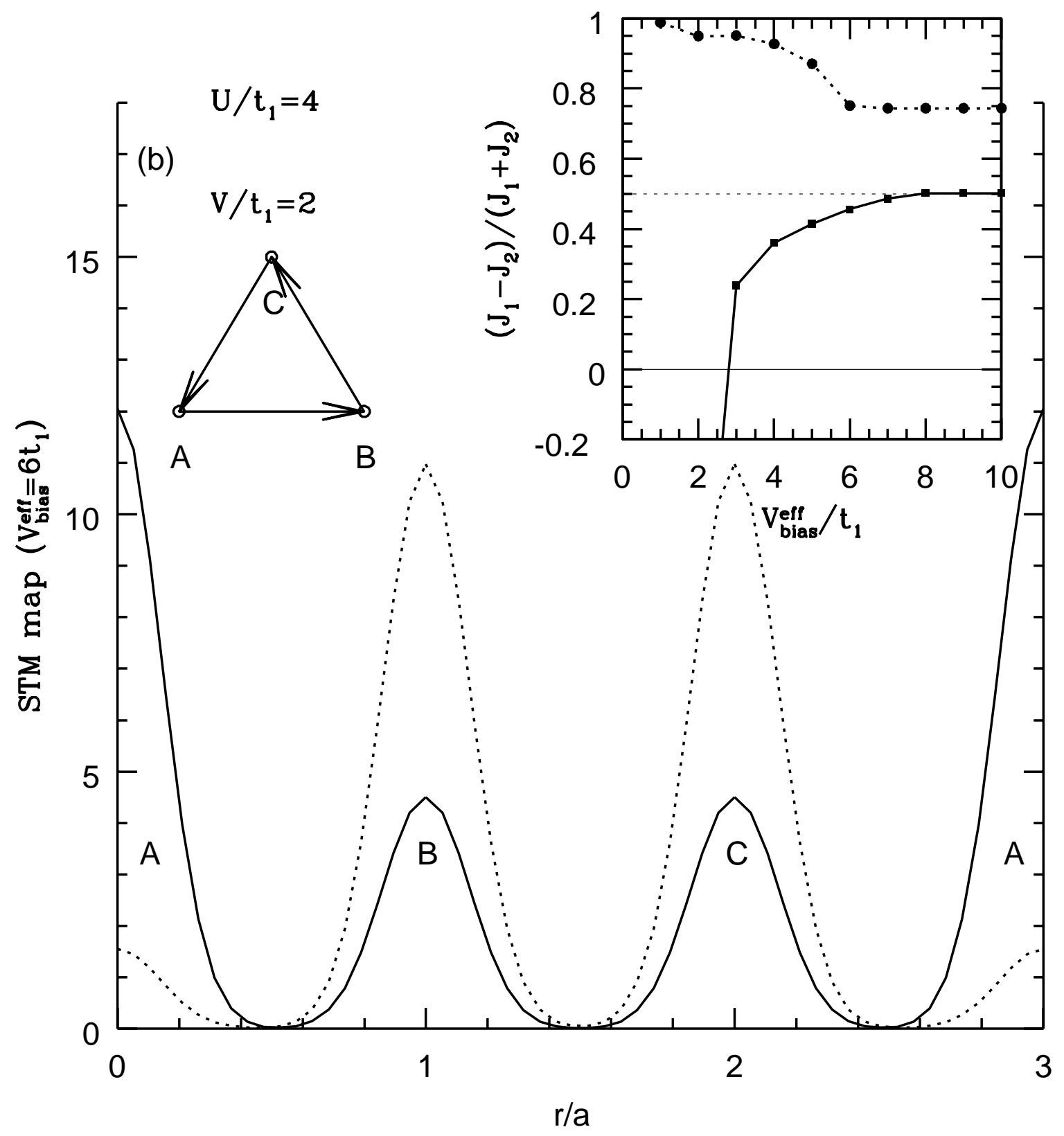

